\title{
7
}

\section{Los valores de la cultura económica cooperativa}

\author{
Dña. Virginia Estarlich \\ Accésit AIDC
}

Respecto al tema referente a los valores que impregnan el cooperativismo, lo he realizado del siguiente modo:

-En principio busqué información relativa a la historia del cooperativismo, el porqué surgen las cooperativas.

-Después me centré en la Cooperativa de Rochdale como máximo exponente del cooperativismo, e impulsora de este movimiento.

- En el trabajo he buscado desarrollar cómo han llegado a la actualidad los valores cooperativos, y cuál ha sido su transformación desde Rochdale.

- He intentado presentar los informes de dos figuras destacadas en el estudio cooperativo, como son: Lars Marcus y Sven Ake Böök, no obstante, he hecho un recorrido por los Congresos más relevantes de la $\mathrm{ACl}$.

- Al mostrar las pautas relativas a cada Congreso, he buscado el enfoque primordial que se da a los valores y a los principios, estableciendo entre ambos un nexo de unión.

- He hecho un estudio de los valores imperantes hoy en día, como se desarrollaron a partir de la Declaración de Manchester de 1995.

- También me he centrado en el desarrollo de los principios cooperativos.

- Por último he buscado encontrar un nexo de unión entre los principios cooperativos y los valores, éstos como fuente de influencia de los principios cooperativos.

- Para finalizar, he intentado extraer una síntesis, y dar una conclusión final del tema, estableciendo las tendencias de los valores cooperativos en el siglo XXI. 
Para poder entrar a analizar los principios y valores que impregnan el cooperativismo es esencial hacerlo desde un punto de vista sociológico e histórico.

Es por ello, que debe hacerse una mención necesaria a la historia, para averiguar cómo y por qué surgen los valores y principios cooperativos.

Una fecha clave en la aparición del cooperativismo propiamente dicho es 1844, con la experiencia cooperativa en Gran Bretaña de Rochdale, donde aparecieron los primeros principios cooperativos que más tarde serían adoptados por el resto de cooperativas y que perdurarán hasta nuestros días.

Sin embargo, las necesidades humanas de aunar esfuerzos, aparecen mucho antes de 1844, aunque no puede hablarse de movimientos cooperativos propiamente dichos, se dan formas de colaboración que presentan ciertas semejanzas con el cooperativismo.

En principio, desde una visión histórica y sociológica, podemos ver que el hombre para satisfacer sus necesidades, aúna esfuerzos con sus semejantes para superar las dificultades, estas asociaciones serían los precedentes o formas rudimentarias de fenómenos cooperativos, las tribus prehistóricas son los fenómenos asociativos que dan comienzo a la agrupación de los seres humanos, puesto que no existe división marcada de funciones en la tribu, y el trabajo está relacionado con el vínculo familiar, la necesidad de supervivencia relacionada con la caza expresa el mejor grado del sistema cooperativo, en el cual todos los miembros varones de la tribu se dedican a cazar conjuntamente, dando lugar a un «especie de cooperativa».

Posteriormente en la Edad Media, los señores feudales, establecen servicios económicos, que van a ayudar a superar algunas dificultades económicas, dando lugar a los municipios donde una agrupación de personas administraban esos municipios, siempre con la inquietud de aunar esfuerzos para la consecución de la satisfacción de unos fines económicos para la mayor parte de la comunidad. Por ello, cabe establecer un fuerte paralelismo entre las creaciones corporativas y gremiales con las modernas cooperativas, puesto que, éstas surgieron para la agrupación de colectivos, con intereses similares. En estas épocas para hablar de cooperativismos debemos analizar el término desde una perspectiva muy amplia del concepto, y por ello, también podríamos encuadrar dentro del cooperativismo, la vida en los monasterios 
donde se regían en comunidad y funcionaban como una especie de cooperativas, en función y beneficio de la propia comunidad.

Como se puede observar durante la historia siempre ha existido en la conciencia del hombre la necesidad de unirse para lograr de una forma viable la consecución de sus fines que de no ser así hubiese sido difícil de conseguir, por ello, también cabe hacer mención a las cofradías de pescadores como una forma de cooperativa, pero siempre como anteriormente se ha dicho tomando la definición de cooperativa en un sentido amplio.

Dentro de la evolución del cooperativismo o de las formas incipientes de cooperativismo, debe hacerse especial mención a Owen y a Fourier, como teóricos que con su doctrina van a crear una serie de proyectos dando lugar a pequeños esbozos de cooperativismo, aunque con una gran carga ideológica y utópica, que desembocan en ambos fracasos, tanto las prácticas de Owen con la «Nueva Armonía», como los falansterios de Fourier.

No sólo estos proyectos fracasan sino que otros intentos cooperativos también lo hacen y sólo a partir de 1844 es cuando podemos hablar de la aparición del cooperativismo con la experiencia de la cooperativa de Rochdale, la cual aparece en Gran Bretaña y esta experiencia va a ser el exponente más notable del cooperativismo, pero también debe tenerse en cuenta que este nuevo movimiento no nace de forma espontánea, sino que recibe influencia de los valores aparecidos con la teorización realizada desde 1820 por los pensadores socialistas premarxistas, desarrollado en Gran Bretaña por Owen, W. Thompson, G. Mudie, W. King. si anteriormente las formas cooperativas surgen espontáneamente sin ningún tipo de ideales, con la única finalidad de defensa de los intereses de los trabajadores, sin ninguna carga ideológica, es a partir de la experiencia de Rochdale, cuando las cooperativas van a tener presente una serie de valores y principios que perdurarán a lo largo de la historia para poder llevar a cabo la consecución de sus fines.

También las cooperativas surgen y en el caso concreto la cooperativa de Rochdale como reacción de los trabajadores a la Revolución industrial y al incipiente sistema capitalista, con la aparición de las cooperativas ante todo se busca la satisfacción de los intereses de los trabajadores, que no ven cubiertas sus necesidades, hito relevante en toda la historia, la búsqueda del ser humano mediante la asociación, de un sistema viable para la consecución de unos determinados fines 
y la satisfacción de sus propias necesidades, encontrando en las cooperativas la solución más viable para conseguir cubrir esas necesidades, que no son cubiertas por otros medios o sistemas.

Cabe analizar cómo surge la cooperativa de Rochdale, y de ella se puede decir que aparece para hacer frente a la negativa de la compañía de gas, para realizar el suministro, y por otro lado la creación de una cooperativa de consumo para combatir el desabastecimiento de alimentos por lo cual, en un principio 28 pioneros (cooperativistas), fundaron esta cooperativa, que con el tiempo se fue ampliando, entre los cooperativistas se encontraban seis discípulos de Owen, que impregnaran con sus ideales los principios que van a dirigir este nuevo fenómeno del cooperativismo; si en un principio con la creación de la cooperativa de Rochdale se buscaba el abastecimiento de gas y el alimenticio, por las necesidades insatisfechas por parte del gobierno como por parte de la empresa suministradora, estos 28 pioneros, van adquiriendo una mayor visión del fenómeno cooperativo para adquirir nuevas empresas y llevar a cabo la consecución de otros fines, y satisfacer de este modo todas sus necesidades, adquiriendo un marcado ideal social.

En lo concerniente a los valores y principios que se desarrollaron en esta época, y que van a ser el eje central para la creación de posteriores cooperativas son: el configurar a las cooperativas como entidades democráticas, los socios serán partícipes de la toma de decisiones en la cooperativa, creando un mayor vínculo cooperativacooperativista.

A pesar de encontrarnos en una época en la cual el valor democracia, era algo que en otros ámbitos de la vida social era difícil de promulgar, las cooperativas asumen este valor primordial como el referente para este tipo de organizaciones, de este valor de democracia, se derivan otra serie de principios como son: la igualdad de derecho a voto, la retribución al capital social obligatorio con un interés limitado y la creación de patrimonio cooperativo irrepartible.

Estos van a ser los principios que van a impregnar la filosofía cooperativista a lo largo de la historia y que van a perdurar hasta nuestros días.

Estos principios de Rochdale son:

1. Control democrático: una persona, un voto.

2. Puerta abierta: libre adhesión (ingreso y retiro voluntarios). 
3. Interés limitado al capital.

4. Retorno de excedentes en proporción a las compras.

5. Ventas al contado.

6. Venta de mercancías de buena clase.

7. Educación de los socios.

8. Neutralidad política y religiosa.

Como puede observarse de estos principios y de los descritos anteriormente, éstos van a ser los adoptados y desarrollados en todos los tipos de cooperativas, siendo válidos para el funcionamiento de cualquier clase de cooperativa adaptándolos a sus necesidades y a su política de funcionamiento.

Posteriormente con la aparición del fenómeno cooperativo, surge en 1895 la Alianza Cooperativa Internacional, que también se hace eco de estos principios unificándolos e identificándolos cada vez más con el movimiento cooperativo, aunque cabe hacer hincapié en la evolución de la sociedad y en la aparición de nuevos valores y principios en un mundo en constante transformación, y con la aparición de nuevas formas y tipos de cooperativas, los primeros principios fueron establecidos para una cooperativa de consumo; es por ello, que se deben de readaptar estos principios a cada época y a cada clase de cooperativa.

Como hemos podido observar el movimiento asociacionista se da a lo largo de la historia, y el hombre busca agruparse para conseguir sus fines y satisfacer sus necesidades.

Pero no sólo el cooperativismo se da de forma única en Gran Bretaña, también surgen movimientos en Francia, Alemania e Italia, algunos de los ideólogos más destacados en estos países en el siglo XIX: J.Ph. Buchez y Ch: Fourier en Francia, Darlehens-Kassenvereineen y V.A: Huber en Alemania; en Italia los promulgadores de las cooperativas de trabajo asociado entre obreros y de crédito entre agricultores, fueron E. Nazzani, L. Luzzati; L. Wollenborg y U. Rabbeno.

Cabe hacer mención de otras dos figuras importantes en lo concerniente al cooperativismo, y éstos son Stuart Mill, que influyó para que se promulgara en 1852, la primera ley en el mundo para regular el fenómeno cooperativo, la Industrial and Provident Societies ACT, posteriormente en 1866, León Walras, funda la revista Le Travail, dedicada al asociacionismo cooperativo. 
De todo lo anteriormente dicho se puede extraer la idea característica que el cooperativismo en un período corto de años se afianza en todos los países, como una nueva fórmula en contra de la Revolución Industrial y el incipiente sistema capitalista, pese a la aparición en todos los países de nuevas corrientes doctrinales, en la creación de cooperativas todas van a tener presentes los principios promulgados por la cooperativa de Rochdale, que anteriormente hemos indicado.

Ante un mundo constantemente en transformación, los principios desarrollados en 1844, toman importancia a lo largo de los años, dado su flexibilidad y la capacidad de adaptación a todas clases de cooperativas y a todas las épocas, puesto que, son fórmulas viables para el desarrollo y la organización empresarial y de funcionamiento de todas las clases de cooperativas en cualquier época.

Teniendo en cuenta la historia y en concreto al tema a tratar, debemos establecer cómo los valores y principios se han desarrollado en el seno del cooperativismo y cómo han ido adaptándose al sistema cooperativista.

En primer lugar debemos determinar cuál ha sido el papel primordial de la Alianza Internacional Cooperativa (ACI), como institución tuteladora del cumplimiento de los principios cooperativos por parte de todas las cooperativas y como ente que vela por la reinterpretación y adaptación de los principios cooperativos en todos los tiempos a todas las clases de cooperativas.

Por ello que para llegar a una conclusión sobre los valores y principios cooperativos en el siglo XXI y su futuro, es pertinente establecer el porqué surgen estos principios y cómo evolucionan a lo largo de la historia del cooperativismo. Siendo desde 1895 la ACl el ente encargado de velar por estos principios y de que sean respetados por todos los cooperativistas.

Es por ello que la $\mathrm{ACl}$ va a ser la encargada desde hace más de 100 años de buscar las fórmulas integradoras de los principios cooperativos a las nuevas necesidades, siempre cambiantes en el mundo.

Siempre teniendo en cuenta la búsqueda de identidad cooperativa y de reformulación e integración de los principios cooperativos desarrollados en Rochdale y que posteriormente adoptó la ACl.

Esta búsqueda de identidad por parte del cooperativismo va a desarrollarse en múltiples Congresos, por ello cabe hacer hincapié en los 
más relevantes, ya que éstos van a ser los que den luz a la reinterpretación y reformulación de los principios cooperativos.

En 1930 en el XIII Congreso de la ACI de Viena, empieza a apuntarse la definición de los principios cooperativos, pero basándose en los desarrollados en la cooperativa de Rochdale, y siendo éstos comunes a cualquier clase de cooperativa, aunque en este Congreso se enuncian una serie de principios no se hace ninguna referencia a los valores, parece impensable en este Congreso que una serie de valores inspiren a los principios cooperativos.

Es en este Congreso cuando la $\mathrm{ACl}$ pasa a ser el ente rector, en materia de principios, que posteriormente dará lugar a la creación de comisiones para realizar los pertinentes informes para el desarrollo e integración de los principios formulados a partir de Rochdale.

Estableciendo en este Congreso siete principios básicos y esenciales, que posteriormente en 1937 en el congreso de París, van a ser desarrollados:

1. Control democrático: una persona, un voto.

2. Puerta abierta: libre adhesión (ingreso y retiro voluntarios).

3. Interés limitado al capital.

4. Retorno de excedentes en proporción a las compras.

5. Ventas al contado.

6. Venta de mercancías de buena clase.

7. Educación de los socios.

Estos siete principios, más un principio «sui generis» como el principio de neutralidad política y religiosa, se establecen como básicos para la constitución de una cooperativa.

También se establece una clara diferenciación y jerarquización de estos principios estableciendo cuatro de ellos como exigibles para la constitución de una cooperativa y tres de los restantes como principios recomendables pero no exigibles.

Como puede observarse, se distingue en este Congreso, los principios por categorías, aunque no se hace mención expresa a los valores que pudiesen inspirar esos principios, aunque se apunta ya algunos conceptos como la equidad, la libertad, la voluntariedad, la universalidad; confundiendo estos valores con los principios, y estableciendo un nexo entre ellos. 
En el Congreso de Bournemonth, no se establecen notas muy significativas, pero sí cabe destacar el énfasis que se ponen en destacar nuevos principios que con el tiempo pasan a ser de los más relevantes, y éstos son: la educación (esbozado en anteriores congresos) y la intercooperación, estos principios se van a integrar en el sistema cooperativista y también se van a readaptar y explicitar viejos principios como el de libre ingreso, intereses al capital y el reparto de excedente, desapareciendo de entre ellos el principio de venta al contado, con ello lo que se pretendía era la universalización de los principios cooperativos a toda clase de cooperativas.

Otra reestructuración importante es la eliminación jerárquica que se había hecho de los principios cooperativos, dando el mismo valor a todos los principios y eliminando el orden establecido en el Congreso de 1930.

Como puede observarse, en un período corto de tiempo la visión respecto de los principios cooperativos tiende a universalizarse y busca la integración de los principios como nexo para la universalidad del cooperativismo, por ello, con la celebración del Congreso de Viena en 1966, es cuando se propugna la visión global de estos principios, marcando esta fecha el punto de inflexión entre la teorización de los principios, y estableciendo una nueva formulación respecto a la reiteradamente y ya mencionada universalización y conjunción de los principios desarrollada por la $\mathrm{ACl}$.

Todo ello quedará desarrollado en el Estatuto de la $\mathrm{ACl}$ y será el eje fundamental para llevar a cabo la caracterización de las cooperativas que quieran ser parte de la $\mathrm{ACl}$.

Pero es en 1980 en el Congreso de Moscú cuando A. F. Laidlaw, presenta en su informe una visión de futuro que perdurará hasta nuestros días y es la búsqueda de la identidad cooperativa y la reconducción de los principios cooperativos al nuevo sistema de mercado.

Dando paso a la fecha clave en el cooperativismo y sobre todo en el tema que nos ocupa y es 1988 en el Congreso de Estocolmo donde por primera vez se va a presentar la noción de valor y su integración en el cooperativismo como pautas inspiradoras de los principios, por ello mención especial debe hacerse a Lars Marcus inspirador del establecimiento de los valores como instrumentos básicos en el contexto del cooperativismo para la revalorización de éste. Lars Marcus introdu- 
jo el término valor y el contenido esencial de estos valores como fuente inspiradora de los principios cooperativos.

Con ello se busca en esta época el establecimiento de una serie de valores que se encargarán de dar sentido a los principios cooperativos, y llevar a cabo una reactivación del proceso cooperativista, aunque si bien es la primera vez que se habla de valores, el tema parece estancarse y tan sólo se señala la necesidad de la existencia de los valores para determinar y dar contenido a los principios cooperativos.

Es en 1992 en el Congreso de Tokio y bajo el informe elaborado por Sven Ake Böök, donde claramente se van a formular los valores que van a impregnar los principios cooperativos, que posteriormente en 1995 serán reformulados y desarrollados.

Este autor para desarrollar los valores que dan sentido al cooperativismo y que se esbozan de forma explícita en 1988, tiene en cuenta la transformación del mercado y la adaptación que debe realizar el sistema cooperativista, dado que el mercado tiende cada vez más a la globalización y a ser más interdependiente, apuntado como valor fundamental para adaptarse el nuevo sistema la solidaridad global como denominador común entre el resto de valores.

En su informe se centra en tres puntos básicos que son:

1. Dar viabilidad a los tradicionales valores cooperativos.

2. Establecer valores globales para el futuro.

3. Dar directrices a cerca de los principios establecidos en la $\mathrm{ACl}$.

Establece tres especies de valores básicos, identificando ideas básicas, ética básica y principios básicos.

Respecto a las ideas básicas establecidas de forma explícita por la $\mathrm{ACl}$ se encontrarían, la igualdad unida al valor de democracia, la equidad, la autoayuda voluntaria y mutua; como valores económicos y sociales la emancipación económica social.

Apuntadas estas ideas básicas cabría establecer la ética que deberían seguir las cooperativas para desarrollar los principios y ésta sería: la honestidad, la ayuda mutua, el pluralismo y la constructividad entendida como confianza en la vía cooperativa.

Como puede observarse, este autor se ocupa de valores individuales que podrían ser considerados cualidades personales, es por ello, 
que establece una serie de valores globales relativos y aplicables a todas las cooperativas y éstos son:

- Asociación de personas.

- Promoción eficiente de los miembros.

- Autonomía e independencia.

- Identidad y unidad.

- Educación.

- Distribución justa de los beneficios- equidad.

- Cooperación nacional e internacional.

Pero no sólo establece esta serie de valores sino como antes hemos expuesto, busca la unión de estos valores individuales con los globales, para que las cooperativas en el desarrollo de sus actividades económicas satisfagan las necesidades de las mayorías y ello se consigue estableciendo las directrices que deben seguir las cooperativas basándose en los valores anteriormente desarrollados y la conjunción de ambos, debe dar lugar a que las actividades económicas deben satisfacer necesidades, y que las cooperativas se consideren organizaciones que realicen actividades económicas para satisfacer esas necesidades, en un marco democrático y participativo, con un mayor desarrollo de los recursos humanos, y una mayor responsabilidad social, y por último la intercooperación, la cooperación nacional e internacional.

A partir de estas directrices y siguiendo el informe de Sven Ake Böök, que acabamos de exponer se llega hasta el Congreso de Manchester de 1995, en este Congreso se busca encauzar el tema relativo a los valores que deben impregnar los principios cooperativos y a su vez se pretende dar vigencia a los principios y adaptarlos a las nuevas tendencias del mercado.

Como hemos visto, la formulación e inclusión de los valores en un Congreso es reciente, pese a que el sistema cooperativista desde sus comienzos se había regido por unos principios, pero no se establecían cuáles eran los valores esenciales o básicos que inspiraban esos principios.

En el Congreso de Manchester de 1995, tras el informe elaborado por lan MacPherson, se adopta el acuerdo que da lugar a la Declaración de la Alianza Cooperativa Internacional sobre la Identidad Cooperativa.

Esta Declaración consta de dos partes, la primera parte como documento principal recoge la definición de cooperativa y el listado de los valores, a su vez también esta primera parte recoge la definición 
de los principios y su enunciado. La segunda parte establece los diferentes extremos seguidos en la Declaración, explicándolos e interpretándolos.

Para desarrollar el tema de los valores y principio a partir de esta Declaración es necesario, fijarnos en la definición que se da sobre la cooperativa, estableciendo qué es una cooperativa:

«Una cooperativa es una asociación autónoma de personas que se han unido de forma voluntaria para satisfacer sus necesidades y aspiraciones económicas, sociales y culturales en común mediante una empresa de propiedad conjunta y gestión democrática».

Siguiendo esta definición se denotan cuáles son los valores y principios que imperan en el cooperativismo, aunque también en la Declaración, se establecen cuáles los valores en que deben basarse las cooperativas, y éstos son:

- Autoayuda.

- Autorresponsabilidad.

- Democracia.

- Igualdad.

- Equidad.

- Solidaridad.

No sólo la declaración enumera estos valores, sino que recoge viejos valores siguiendo la tradición de sus fundadores, enumerando los siguientes valores, los socios cooperativos hacen suyos los valores éticos de la honestidad, la transparencia, la responsabilidad y la vocación social.

Al analizar el tema de los valores no cabe establecer una jerarquía entre ellos, sino que lo ideal para encontrar la identidad cooperativa es agruparlos, puesto que los valores éticos pueden servir de enlace con otros tipos de organizaciones imperantes en el mercado económico y que adoptan también los valores éticos de honradez, transparencia, responsabilidad social y vocación social.

Tanto unos valores como los otros deben complementarse, aunque los valores de autoayuda, autorresponsabilidad, democracia, igualdad, equidad y solidaridad, serían los valores operativos que deben inspirar los principios cooperativos.

Cabe dar significado a estos valores que dan sentido al cooperativismo si se tiende al asociacionismo, el individuo debe en la 
cooperativa realizar las acciones que sean mejores para la entidad a la que pertenece, implicándose al máximo y responsabilizándose de cada acción que realice en función del interés cooperativista, fomentado la educación y la integración de nuevos socios.

Un valor básico y fundamental dentro de las cooperativas es el valor de la igualdad, reflejado en la unidad de los cooperativistas, desarrollado en los derechos de participación, información y el derecho a ser escuchados.

Junto al valor de la igualdad se establece el valor de la equidad refiriéndose al trato equitativo que debe dispensarse a los socios en relación a la forma de recompensar su participación en la cooperativa, esta recompensa al cooperativista viene determinada mediante retornos, reducciones de precios o bien reservas de capital en su nombre.

Pero un valor que engloba a los anteriores es el valor de la solidaridad, el cual establece la máxima a seguir por todos los cooperativistas, por el cual la asociación es preponderante al individualismo, esta solidaridad es el valor para mantener unidos a los cooperativistas y con ello lograr un mayor beneficio y satisfacción de sus necesidades.

Solidaridad entendida como fórmula perfecta de unión entre todos los valores, pues, la solidaridad englobaría tanto los valores individuales como la autoayuda y la autoresponsabilidad con el valor de la Intercooperación, el cual hace referencia a la cooperación y asociación de cooperativas.

Teniendo en cuenta siempre esta serie de valores, debemos de fijarnos en la Declaración al determinar "Siguiendo la tradición de sus fundadores, los socios cooperativos hacen suyos los valores éticos de la honestidad, la transparencia, la responsabilidad y la vocación social».

Cabe establecer una cierta conexión entre los valores propios de las cooperativas y los valores que acabamos de enunciar puesto que, éstos pueden darse en cualquier tipo de organización, con ellos, se busca dar un significado equitativo al sistema de mercado, pero en las empresas cooperativas estos valores éticos tienen mayor relevancia dado que, en este tipo de organizaciones se busca el bienestar de los socios y valores como la honestidad, la transparencia, la responsabilidad y la vocación social son los que impregnan al cooperativismo de un mayor vínculo de compromiso, tanto de los socios en beneficio de 
la cooperativa, como de las personas encargadas de gestionar dicha cooperativa, siempre teniendo en cuenta estos valores superiores para el buen funcionamiento y fin esencial de la cooperativa que es servir a sus socios y cubrir las necesidades de éstos, dado que éste sería el fin con el que se crea cualquier cooperativa.

Por ello siguiendo a G. Laserre, en relación a los valores morales que deben imperar en el cooperativismo, y que dan sentido a este movimiento son: el valor del factor humano y el respeto a cada individuo, dando sentido a la igualdad, y sentido a la dignidad en el trabajo, centrándose en la autoayuda y en la autoresponsabilidad, como valores esenciales que darán lugar a la solidaridad, como manifestación de puesta en común de esfuerzos, como de reparto equitativo de las ganancias obtenidas, etc.

Con la promulgación de estos valores se busca que los socios sean responsables de sus actos no solamente en el seno de la cooperativa, sino que los hagan suyos en todas sus acciones.

Si hasta ahora nos hemos ocupado de los valores, debemos establecer cómo se materializan esos valores, por ello, tenemos que hacer especial referencia a los principios cooperativos como pautas de comportamiento para un mejor desarrollo y funcionamiento del cooperativismo.

Volviendo a los orígenes de la aparición de los principios cooperativos, desarrollados en la Cooperativa de Rochdale en 1844, sus cooperativistas formulan una serie de directrices que van a perdurar hasta nuestros días, aunque como se ha estado constantemente repitiendo, estos principios, han sido revisados y reformulados a lo largo de la historia por su órgano tutelador, la $\mathrm{ACl}$, atendiendo siempre al ideal de búsqueda de la identidad cooperativa, aunque también en el seno de la $\mathrm{ACl}$ y en todos sus Congresos se ha intentado equilibrar estos principios a las necesidades imperantes en cada momento, pero siempre teniendo en cuenta los principios desarrollados en Rochdale y tendiendo siempre a la universalización de ellos.

En la Declaración de Manchester de 1995, documento recienten más importante para todas las cooperativas, es donde mejor puede verse la búsqueda de universalización de los principios cooperativos, para que puedan ser viables no sólo a cualquier tipo de cooperativa, sino que también puedan ser desarrollados en el seno de una cooperativa en cualquier país, independientemente del lugar donde sean constituidas. 
Es por ello, que los principios cooperativos desarrollados en la Declaración de Manchester de 1995, son las directrices o mandamientos que deben seguir los cooperativistas para el desarrollo y mejor funcionamiento de la cooperativa.

Como anteriormente hemos indicado al establecer los valores que van a regir el cooperativismo y que influirán en los principios, hay que buscar su nexo o lazo de unión entre ellos.

Atendiendo a la definición que se hace en la Declaración de Manchester sobre los principios, podemos saber qué se entiende por principios y cuáles van a ser esos principios.

La Declaración establece qué se entiende por principios:

«Los principios cooperativos son pautas mediante las cuales las cooperativas ponen en práctica sus valores».

Y cuáles son esos principios:

- ADHESION VOLUNTARIA Y ABIERTA.

- GESTION DEMOCRATICA POR PARTE DE LOS SOCIOS.

- PARTICIPACION ECONOMICA DE LOS SOCIOS.

- AUTONOMIA E INDEPENDENCIA.

- EDUCACION, FORMACION E INFORMACION.

- COOPERACION ENTRE COOPERATIVAS.

- INTERES POR LA COMUNIDAD.

Estos van a ser los principios básicos que van a regir cualquier cooperativa, son las pautas o mandamientos para evaluar el comportamiento y llevar a cabo la toma de decisiones en el seno de la cooperativa, como puede observarse son unos principios tendentes a la universalización, para que cualquier clase de cooperativa pueda adoptarlos, y también cabe decir que aunque han sido readaptados, no difieren de los primeros principios desarrollados en Rochdale.

Relativo a estos siete principios cabe decir que los tres primeros principios, Adhesión voluntaria y abierta; Gestión Democrática por parte de los Socios y Participación económica de los Socios, están dirigidos a la dinámica interna y típica de cualquier cooperativa, mientras que los restantes, Autonomía e Independencia; Educación, Formación e Información, Cooperación entre Cooperativas y Interés por la Comunidad, se refieren tanto a las relaciones dentro como fuera de la cooperativa, son pautas de conducta para la vida cooperativa. 
El primer principio relativo a la Adhesión voluntaria y abierta, debe entenderse como la participación voluntaria de cada persona a integrarse o no en una cooperativa.

Este principio estaría inspirado en los valores de igualdad, autoayuda y autoresponsabilidad.

Este valor debe ser entendido como libertad que tiene cada persona de formar parte de una cooperativa; la expresión "abiertas» hace referencia a todas las personas capaces de utilizar los servicios que ofrece la cooperativa y que estén dispuestas a aceptar las responsabilidades de ser socio, sin discriminación por sexo, razón social, racial, política o religiosa.

Como puede observarse con este principio se busca la integración en el seno de la cooperativa estableciendo un orden superior, en el cual el buen funcionamiento de la cooperativa debe estar por encima de cualquier tipo de discriminación, siendo este principio la base democrática en la cual se recogería por excelencia el valor fundamental de la igualdad, al establecer una fuerte restricción a la discriminación de cualquier tipo.

El segundo principio referente a la Gestión Democrática por parte de los socios, establece que «las cooperativas son organizaciones gestionadas democráticamente por los socios, los cuales participan activamente en la fijación de sus políticas y en la toma de decisiones».

Este derecho quizás sea el más controvertido, aunque no siempre los dirigentes elegidos cumplen con las expectativas que los socios habían depositados en ellos; sin embargo, atendiendo a este principio se puede observar cómo se da plenamente el principio de igualdad, puesto que los socios cuentan todos con los mismos derechos de voto, y de información. Con la regla democrática un hombre un voto, se establece un mayor contacto entre el cooperativista y la cooperativa, dado que el cooperativista se involucra más en la vida y funcionamiento de la cooperativa.

El tercer principio hace referencia al sistema económico y se fundamenta en el valor de la equidad, dado que se establece que «los socios contribuyen equitativamente al capital de sus cooperativas y lo gestionan de forma democrática», este principio describe cómo participan los socios en el capital de las cooperativas y la forma en que deciden distribuir los excedentes. 
De este principio se deriva el poder que tienen los socios de gestionar el capital de sus cooperativas, siendo los que toman las decisiones de cómo va a repartirse ese capital.

El cuarto principio Autonomía e independencia, este principio es la máxima a desarrollar en cualquier cooperativa, dado que, tiende a la necesidad de cualquier cooperativa a ser autónoma, ya desde el desarrollo de los primeros principios aparece este principio como señal de identidad del sistema cooperativista, con ello se busca la autonomía de la cooperativa tanto a nivel político como a nivel religioso. Esta autonomía por parte de la cooperativa es un claro reflejo del sistema democrático imperante en el cooperativismo.

Ante la incipiente colaboración de las cooperativas con otras organizaciones económicas, debe tenerse en cuenta que cada vez cabe tener arraigado este principio básico e identificativo de las cooperativas, que tienen como único fin la satisfacción de las necesidades de sus socios.

El principio de Educación, Formación e Información, se presenta como una alternativa viable para la cultura cooperativista.

Se tiende cada vez más a dar una educación y formación en el seno de la cooperativa, tanto a los socios como a sus dirigentes, con ello se pretende asegurar y formar de la mejor manera posible a todos los individuos que formen parte de la cooperativa, para que hagan posible las tareas que les son encomendadas, y satisfacer las necesidades de los socios en la mayor medida posible.

El sexto principio, establece la cooperación entre cooperativas, es un principio nuevo y se formula atendiendo a las nuevas necesidades del mercado tendente a la globalización y a la creación de las cooperativas de segundo grado.

Teniendo en cuenta como se ha establecido anteriormente la autonomía de las cooperativas, cada vez más se producen más interconexiones entre cooperativas y éstas a su vez con otros órganos económicos.

Con este principio basado en la solidaridad, se buscan apoyos para que las cooperativas se revaloricen.

Por ello, las cooperativas deben cooperar en todos los sectores de su actividad; potenciar las estructuras federativas; adecuar la legisla- 
ción hacia los nuevos cambios y necesidades; buscar medios de financiación a través de los bancos cooperativos y por último formar de la mejor forma posible a sus socios y dirigentes, utilizando la tecnificación y modernización necesaria.

El último principio relativo al interés por la comunidad, es la responsabilidad de los socios a trabajar por la comunidad, este principio se engloba en las necesidades estructurales de cada comunidad, puesto que las cooperativas suelen surgir en el seno de las comunidades como entes de satisfacción económica. Hoy en día el interés por la comunidad queda reflejado en la protección y el desarrollo del medio ambiente.

En grandes líneas éstos serían los principios que imperan en el cooperativismo y son las pautas que deben seguir todas las cooperativas.

Al describir estos principios nos encontramos que se trata de unas directrices muy simples, las cuales se pueden aplicables a cualquier clase de cooperativa, independientemente del lugar donde se halle ubicada. Son unos principios prácticos, flexibles y en suma aplicables a cualquier clase de cooperativa.

En la Declaración de Manchester lo que se ha buscado es que la universalización de los principios cooperativos se adapte a todas las clases de cooperativas, y que estos principios tengan viabilidad en un futuro. Lo primordial en esta Declaración es que los principios cooperativos sean claro referente de la identidad cooperativa.

En conclusión, cabe destacar que ante todos los acontecimientos acaecidos en el transcurso de la historia, tanto en el plano político, económico y social, tiene claro reflejo en la sociedad que busca nuevas fórmulas para cubrir sus necesidades, es por ello, que los valores cooperativos deben tender al servicio de los cooperativistas y se debe reivindicar éstos, a través de las organizaciones cooperativas, como instrumentos de defensa y protección del ser humano, reflejado en el arraigado sistema democrático que impera en la concepción y funcionamiento de las cooperativas.

Es por ello, que las cooperativas deben estar preparadas para todos los cambios e introducirse en todas las áreas de mercado, puesto que cada vez se tiende a la globalización, las cooperativas deben establecerse como entidades económicas con unos valores superiores, para la consecución de todos sus fines. 
Con el asentamiento de unos valores firmes que inspiren a unos principios universales para todas las cooperativas, es cuando realmente se podrá alcanzar la identidad cooperativa.

Aunque también debe tenerse en cuenta que ante todo una cooperativa es una empresa y debe buscar un nexo de unión para conjugar los valores cooperativos con los valores económicos, estos dos aspectos deben de complementarse y potenciarse mutuamente, puesto que, si una cooperativa fracasa como empresa económica, fracasa también su pretendida proyección social y humana, llevando al descrédito a la institución. Y ello cabe resumirse en una frase, los valores morales se pierden sepultados por los económicos (José Luis Aranguren).

Por ello el futuro de las cooperativas pasa por adoptar junto a sus valores, los valores imperantes en el sistema económico, para seguir funcionando, pero la adopción de esos valores de la mejor manera para que se dé la identidad cooperativa.

\section{Bibliografía}

LUIS y NAVAS, Jaime: «Derecho de coopeativas». Bosch.1972.

Principios cooperativos: sextas jornadas cooperativas, 1988, Asociación de Expertos Cooperativas (AEC).

Declaración de la Alianza Cooperativa Internacional sobre la Identidad Cooperativa.1995.

Artículos:

AKE BÖÖK; Sven, Cooperativas valores fundamentales y principios cooperativos, CIRIEC.1990.

ARANZADI, Dionisio. "Actualidad de los valores y de la formación en el cooperativismo». Anuario de estudios cooperativos.1989.

MARTINez ChARTERINA, Alejandro. "Los valores y principios cooperatiVOS». REVESCO:1995.

MARCus, Lars. "Cooperativas y valores básicos». Ponencia. Boletín de estudios y documentación.

GracognA, Dante. «Reflexiones sobre los valores y principios en la alianza cooperativa internacional». Anuario de estudios cooperativos.1991.

SANZ JARQUE, Juan José. "La función de las cooperativas en el siglo XXI: valores y principios». Anuario de estudios cooperativos.1995. 